\title{
Ganho de Peso Gestacional e Fatores Associados em Gestantes e Recém-Nascidos
}

\author{
Rafaela Santi Dell'Osbel ${ }^{1}$, Cleber Cremonese ${ }^{2}$, Maria Luisa de Oliveira Gregoletto ${ }^{3}$
}

\begin{abstract}
RESUMO
Objetivo: O presente estudo teve como objetivo medir o Ganho de Peso Gestacional (GPG) e identificar os fatores associados em gestantes e recém-nascidos. Métodos: Trata-se de um estudo epidemiológico observacional transversal, em uma coorte constituído por gestantes e recém-nascidos usuários da Atenção Básica de Caxias do Sul/RS. Os dados foram coletados em três momentos distintos, sendo no primeiro e terceiro trimestres gestacional e no primeiro mês após o nascimento do bebê. Resultados: A amostra constituiu-se de 47 gestantes e recém-nascidos. Destas, 28,3\% apresentaram GPG insuficiente, 34,8\% GPG adequado e $37 \%$ GPG excessivo. As gestantes com escolaridade até 10 anos de estudo apresentaram maior prevalência de GPG insuficiente (48,0\%), já nas que dispunham de 11 anos ou mais de estudo foi constatada maior prevalência de GPG adequado $(52,4 \%)(p=0,024)$. Ainda, observa-se associação significativa com comprimento ao nascer ( $p$-valor 0,035$)$ e com a alimentação na primeira semana de vida ( $p$-valor 0,037 ). Conclusões: Conclui-se que as gestantes apresentam elevada prevalência de GPG excessivo. Ainda, identificou-se associação significativa entre o GPG insuficiente com a baixa escolaridade e ao menor comprimento ao nascer. Houve associação entre o GPG excessivo e elevada prevalência para Aleitamento Materno Exclusivo (AME) na primeira semana de vida. Desta forma, percebe-se a necessidade de estratégias para adequar e orientar o GPG, assim como instruir hábitos de vida saudáveis e reforçar a necessidade do cuidado pré-natal para a saúde da gestante e do recém-nascido.
\end{abstract}

Palavras-chave: Gravidez. Ganho de peso. Fatores de risco. Recém-nascido.

$$
\text { GESTIONAL WEIGHT GAIN AND FACTORS ASSOCIATED WITH PREGNANT AND NEWBORNS }
$$

\begin{abstract}
Objective: The present study aimed to measure gestational weight gain (GWG) and to identify the associated factors in pregnant and newborns. Methods: This is an observational cross-sectional epidemiological study in a cohort of pregnant and newborn users of Basic Care in Caxias do Sul/RS. The data were collected in three different moments, being in the first and third gestational trimester and in the first month after the baby's birth. Results: The sample consisted of 47 pregnant and newborns, of whom $28.3 \%$ had insufficient GWG, $34.8 \%$ had adequate GWG and $37 \%$ had excessive GWG. Pregnant women with schooling up to 10 years of schooling presented a higher prevalence of insufficient GWG (48.0\%), while those with 11 years or more of study had a higher prevalence of adequate GWG $(52.4 \%)(p=0.024)$. Also, a significant association with length at birth ( $p$-value 0.035 ) and feeding during the first week of life ( $p$-value 0.037$)$ was observed. Conclusions: It is concluded that pregnant women have a high prevalence of excessive GWG. Also, a significant association was identified between the poor GWG with the low level of education of the pregnant woman and the shorter length at birth. There was an association between excessive GWG and high prevalence for exclusive breastfeeding in the first week of life. Thus, the need for strategies to adjust and guide GWG, as well as to instruct healthy life habits and to reinforce the need of prenatal care for the health of the pregnant woman and the newborn, is perceived.
\end{abstract}

Keywords: Pregnancy. Weight gain. Risk factors. Newborn.

RECEBIDO EM: 13/9/2018

MODIFICAÇÕES REQUERIDAS EM: 15/7/2019

ACEITO EM: 31/7/2019

\footnotetext{
Mestranda pelo Programa de Pós-Graduação em Ciências da Saúde: Ginecologia e Obstetrícia pela Universidade Federal do Rio Grande do Sul (UFRGS). ra.fasanti@hotmail.com

Doutor em Ciência - Saúde Pública e Meio Ambiente pela Escola Nacional de Saúde Pública Sergio Arouca (ENSP/Fiocruz). Mestre em Saúde Coletiva pela Universidade do Vale do Rio dos Sinos (Unisinos). Docente do Programa Integrado em Saúde Ambiental e do Trabalhador, Instituto de Saúde Coletiva (ISC), Universidade Federal da Bahia (UFBA). clebercre@yahoo.com.br

${ }^{3}$ Mestre em Saúde Coletiva pela Universidade do Vale do Rio dos Sinos (Unisinos). Docente do curso de Nutrição na FSG - Centro Universitário (FSG).maria.gregoletto@fsg.edu.br
} 


\section{INTRODUÇÃO}

A gestação é uma fase fisiológica importante ao ciclo vital que envolve uma série de mudanças hormonais e, por isso, tem demanda de cuidados no âmbito da saúde, especialmente o nutricional (WEISSGERBER; WOLFE, 2006). No campo da nutrição essa fase requer cuidados e atenção em razão da variação no metabolismo de nutrientes e aumento na demanda energética da gestante (COUNCIL, 2009). Como a não adequação da alimentação pode acarretar em um aumento dos riscos durante a gestação, cabe ao profissional nutricionista orientar sobre a ingestão correta dos alimentos, quando recomenda-se que as gestantes tenham uma alimentação variada, seguindo as quantidades indicadas pelos guias alimentares (VOIDAZAN et al., 2018).

Para a determinação da necessidade nutricional da gestante é necessário considerar fatores relevantes, como o estado nutricional pré-gestacional, comportamento e estilo de vida, fatores genéticos associados, além de práticas alimentares culturais (COUNCIL, 2009). Como consequência de uma nutrição adequada, seguindo as necessidades individuais de cada gestante, o resultado é o desenvolvimento e crescimento fetal e gestacional adequados (COUNCIL, 2009; DREHMER et al., 2013; HIROOKA-NAKAMA et al., 2018; NUCCl et al., 2018).

O peso gestacional é um determinante para uma gravidez saudável. Nesse sentido, o ganho de peso gestacional (GPG) excessivo ou insuficiente é considerado fator de risco para a gestante e também para o feto (BRASIL, 2011 ; COUNCIL, 2009; DREHMER et al., 2013; NUCCl et al., 2018; ORNAGHI et al., 2018). Assim, torna-se necessário uma avaliação nutricional correta, visando a adequar o GPG, promovendo a saúde materno e infantil e estimulando o crescimento fetal adequado (BRASIL, 2011) .

O cuidado com o recém-nascido é de extrema importância para uma vida futura saudável. Por isso, o acompanhamento durante a gestação é essencial e torna possível a detecção de dificuldades e riscos, facilitando o tratamento e contribuindo para a redução da mortalidade infantil evitável. No Brasil há diversos projetos que priorizam um bom atendimento $\mathrm{e}$ acompanhamento materno-infantil, quando os cuidados com a saúde do recém-nascido começam desde a gestação, visando a garantir saúde e qualidade de vida futura, reduzindo a morbimortalidade infantil (BRASIL, 2011).
Segundo estudos, o GPG excessivo está associado a desfechos negativos, como a macrossomia fetal ou alto peso ao nascer, altas taxas de cesarianas e internações, além de estar relacionado com obesidade e outras doenças na vida adulta (HIROOKA-NAKAMA et al., 2018; NUCCl et al., 2018). Já o GPG insuficiente está associado ao baixo peso ao nascer e prematuridade (ORNAGH et al., 2018). Ainda assim, estudos mostram que o GPG está relacionado ao peso ao nascer do recém-nascido, e o mesmo está relacionado com a mortalidade infantil. Assim, é de extrema importância dar a atenção necessária ao estado nutricional materno e ao ganho de peso durante a gestação, sendo uma fase determinante da saúde na vida adulta (BRASIL, 2011 ; HIROOKA-NAKAMA et al., 2018; NUCCl et al., 2018; ORNAGHI et al., 2018).

Em razão do problema, existem riscos relacionados ao GPG insuficiente ou excessivo tanto para a gestante quanto para o recém-nascido. Sendo assim, verifica-se a necessidade de novas pesquisas dirigidas a esta população, em virtude dos poucos estudos encontrados em âmbito nacional e regional. O presente estudo, portanto, teve como objetivo medir o ganho de peso gestacional e identificar os fatores associados em gestantes e recém-nascidos atendidos na Atenção Básica de Saúde de Caxias do Sul/RS.

\section{MATERIAIS E MÉTODOS}

\section{Delineamento e População de Estudo}

Trata-se de um estudo epidemiológico observacional transversal em uma coorte de gestantes, recorte do Projeto de Pesquisa "Comportamentos de risco à saúde da gestante e o impacto na saúde materna e do recém-nascido na Atenção Básica de Saúde de Caxias do Sul-RS".

\section{Característica Amostral}

A cidade de Caxias do Sul dispõe de 46 Unidades Básicas de Saúde (UBS). Como critério de seleção da amostra foram sorteadas 16 UBS que dispunham de equipes de Estratégia de Saúde da Família. Foram convidadas a participar do estudo as gestantes inscritas no SIS-PRÉ-NATAL na UBS sorteada, com riscos reduzidos no período gestacional, idade igual ou superior a 18 anos e com $\leq 13$ semanas de gestação. Gestantes analfabetas, com idioma de origem diferente do português, com menos de 18 anos e que apresentavam gestação de risco, não foram incluídas no estudo. 


\section{Coleta de Dados}

A coleta ocorreu em três momentos: o primeiro momento (T1) no primeiro trimestre gestacional, o segundo (T2) no terceiro trimestre gestacional e o terceiro (T3) no primeiro mês após o nascimento. Em T1 (julho a outubro de 2017) e T2 (novembro de 2017 a fevereiro de 2018) realizou-se entrevistas com as gestantes e coleta de dados de prontuário. Em T3 (fevereiro a abril de 2018) fez-se a coleta de dados do recém-nascido por meio de um questionário e de dados do prontuário referentes ao nascimento.

\section{Instrumento para Coleta de Dados}

Utilizou-se três questionários padronizados, pré-codificados, elaborados pelos pesquisadores. Realizou-se um estudo piloto com cinco gestantes de uma UBS sorteada, as quais não foram inclusas na amostra final. Ressalta-se que uma parte de dois questionários (T1 e T2), composta por algumas variáveis comportamentais, foram obtidas por intermédio de um questionário autoaplicável.

O primeiro questionário (T1) foi constituído por questões socioeconômicas e demográficas, comportamentais, antropométricas e de hábitos alimentares. As variáveis como idade gestacional, data provável do parto, exames laboratoriais, histórico familiar e pessoal, antecedentes ginecológicos e obstétricos e dados antropométricos, foram coletadas por meio de dados de prontuário.

Referente aos aspectos socioeconômicos e demográficos investigados no primeiro trimestre gestacional (Tabela 1), foram estudadas as seguintes variáveis: idade ( $\geq 27$ e $\leq 26$ ), estado civil (casada/união estável e solteira/separada/divorciada), cor de pele autodeclarada (outras e branca), escolaridade ( $\leq 10$ anos e $\geq 11$ anos), trabalho remunerado (sim e não) e nível socioeconômico (de $\mathrm{A}$ a $\mathrm{B}$, de $\mathrm{C}$ a D, e E).

Ainda, no primeiro questionário ( $\mathrm{T} 1)$, investigou-se histórico de aborto (sim e não) e gestações anteriores (Nenhuma, de 1 a 2, e 3 ou mais) (Tabela 2). Também investigou-se dados como o peso pré-gestacional e a estatura, que foram coletados diretamente do prontuário. Estes dados foram utilizados para calcular o Índice de Massa Corporal (IMC) pré-gestacional, sendo classificado em: baixo peso $\left(<18,5 \mathrm{~kg} / \mathrm{m}^{2}\right)$, adequado $\left(18,5 \mathrm{~kg} / \mathrm{m}^{2},-24,9 \mathrm{~kg} / \mathrm{m}^{2}\right)$, sobrepeso $(25,0$ $\left.\mathrm{kg} / \mathrm{m}^{2}-29,9 \mathrm{~kg} / \mathrm{m}^{2}\right)$ e obesidade $\left(\geq 30,0 \mathrm{~kg} / \mathrm{m}^{2}\right)$. Neste estudo, a variável IMC foi categorizada como dicotômica em eutróficas e não eutróficas.
No segundo questionário (T2) investigou-se a variável de estilo de vida (Tabela 3 ) praticar atividade física semanalmente (sim e não) (WHO, 2010). Referente às variáveis comportamentais foram estudados (Tabela 3): hábito de realizar café da manhã (sim e não), hábito de beliscar entre as refeições (sim e não), insatisfação corporal e sintomas depressivos.

A insatisfação corporal foi avaliada por meio do Body Shape Questionnaire (BSQ), em sua versão em português (CORDÁS; CASTILHO, 1994), permitindo identificar o grau de insatisfação corporal conforme o somatório de pontos (CORDÁs; CASTILHO, 1994). Categorizou-se a variável em ausência ( $\leq 80$ pontos) e presença ( $>80$ pontos) de insatisfação corporal. Identificou-se, também, a presença de sintomas depressivos por meio do Patient Health Questionnaire (PHQ-9), um questionário padronizado e traduzido, composto por nove perguntas. $O$ resultado se dá por meio do somatório dos pontos; assim, categorizou-se a variável em ausência de sintomas ( $\leq 4$ pontos), sintomas leves (5-9 pontos) e sintomas moderados/severos ( $\geq 10$ pontos) (Tabela 3).

Quanto às informações do nascimento e do recém-nascido (T3), as variáveis estudadas foram: idade gestacional do nascimento (pré-termo ( $\leq 37$ semanas), termo (38 a 42 semanas) e pós-termo ( $>42$ semanas)), Apgar em 5 minutos ( $\leq 7$ pontos, $\geq 8$ pontos), peso ao nascer $(<2.500 \mathrm{~g}$ - baixo peso, $2.500 \mathrm{~g}$ a $3.999 \mathrm{~g}$ - peso normal e $\geq 4.000 \mathrm{~g}$ - macrossomia), comprimento ao nascer $(\leq 47 \mathrm{~cm}$ e $\geq 48 \mathrm{~cm})$, perímetro cefálico $(\leq 33 \mathrm{~cm}$ e $\geq 34 \mathrm{~cm}$ ) e tipo de parto (normal ou cesárea). Investigou-se, ainda, a ocorrência de internações com o recém-nascido ( $>72$ horas após o nascimento) (sim e não) e a forma de alimentação do recém-nascido na primeira semana de vida (Aleitamento Materno Exclusivo (AME) e Aleitamento Misto (aleitamento materno e fórmulas infantis)/Fórmulas Infantis/Outros) (BRASIL, 2011) .

\section{Variável Desfecho - Ganho de peso gestacional}

O GPG foi mensurado por meio de dados coletados no prontuário da gestante, no qual diminui-se o último registro de peso gestacional pelo peso pré-gestacional (peso final - peso pré-gestacional), obtendo-se, assim, o GPG. Este foi classificado segundo o IMC pré-gestacional da gestante (baixo peso, adequado, sobrepeso e obesidade), e, desse modo, as gestantes com IMC pré-gestacional adequado podem ganhar de $11 \mathrm{~kg}$ a $16 \mathrm{~kg}$ durante a gestação, mantendo o GPG adequado. Já as gestantes com baixo peso, sobrepeso e obesidade, podem ganhar respectivamente de $12,5 \mathrm{~kg}$ a $18 \mathrm{~kg}, 7 \mathrm{~kg}$ a $11,5 \mathrm{~kg}$ e $5 \mathrm{~kg}$ a $9 \mathrm{~kg}$ durante a 
gestação. Dessa forma, seguindo as classificações do IMC, as gestantes que ganharam peso dentro das recomendações foram classificadas como com GPG adequado; já as que ganharam abaixo e acima dos valores recomendados foram classificadas como com GPG insuficiente e GPG excessivo, respectivamente (COUNCIL, 2009).

\section{Análises de Dados}

A estruturação do banco de dados e análise estatística foram realizadas por meio do programa SPSS Statistic Data 23, e as análises bivariadas foram feitas aplicando-se teste de Qui-Quadrado para comparação de variáveis categóricas, gerando as prevalências para o desfecho e $p$-valor. Considerou-se um nível de significância de $5 \%(p \leq 0,05)$ para identificar associação entre o desfecho e as variantes de exposição.

\section{Aspectos Éticos}

O projeto de pesquisa foi aprovado por um Comitê de Ética em Pesquisa, sob parecer no 2.184.991. Todas as gestantes participantes do estudo assinaram o Termo de Consentimento Livre e Esclarecido.

\section{RESULTADOS}

Foram incluídas no primeiro momento do estudo 76 gestantes; no segundo momento 5,3\% tiveram ocorrência de aborto, 13,1\% foram residir em outras cidades, 9,2\% continuaram o acompanhamento pré-natal por convênio de saúde e 10,5\% recusaram-se a continuar na pesquisa. A amostra final constituiu-se de 47 gestantes e recém-nascidos da Atenção Básica de Saúde de Caxias do Sul/RS; destas, 28,3\% apresentaram GPG insuficiente, 34,8\% GPG adequado e $37 \%$ GPG excessivo. Em relação à idade, encontrou-se uma média de 26,5 anos $( \pm 6,08 \mathrm{DP})$, variando de 18 a 41 anos. Quanto à cor da pele, nota-se que prevaleceu outras (55,3\%). Ainda, observou-se que $68,1 \%$ das gestantes estavam casadas ou em união estável, $55,3 \%$ estudaram até 10 anos e 57,4\% referiram dispor de trabalho remunerado. No que diz respeito à renda, $6,4 \%$ encontravam-se classificadas com nível socioeconômico de A a B (Tabela 1).

Em relação ao GPG, observou-se, de forma significante, que as gestantes com escolaridade até 10 anos de estudo apresentaram maior prevalência de GPG insuficiente (48,0\%), já as que dispunham de 11

Tabela 1 - Variáveis demográficas, socioeconômicas e de moradia em relação ao ganho de peso gestacional em gestantes atendidas na Atenção Básica de Saúde de Caxias do Sul-RS. 2017 (n=47)

\begin{tabular}{|c|c|c|c|c|c|}
\hline Variáveis de exposição & n (\%) & $\begin{array}{l}\text { Prevalência } \\
\text { de GPG* } \\
\text { Insuficiente }\end{array}$ & $\begin{array}{c}\text { Prevalência de } \\
\text { GPG* }^{*} \\
\text { Adequado }\end{array}$ & $\begin{array}{c}\text { Prevalência de } \\
\text { GPG* } \\
\text { Excessivo }\end{array}$ & $\mathrm{p}$-valor** \\
\hline Idade (anos) & & & & & 0,669 \\
\hline$\leq 26$ & $21(44,7)$ & 28,6 & 28,6 & 42,9 & \\
\hline$\geq 27$ & $26(55,3)$ & 28,0 & 40,0 & 32,0 & \\
\hline Estado Civil & & & & & 0,272 \\
\hline Casada/União Estável & $32(68,1)$ & 31,3 & 37,5 & 31,3 & \\
\hline $\begin{array}{l}\text { Solteira/Separada/ } \\
\text { Divorciada }\end{array}$ & $15(31,9)$ & 21,4 & 28,6 & 50,0 & \\
\hline Cor da pele & & & & & 0,787 \\
\hline Outras & $26(55,3)$ & 30,8 & 26,9 & 42,3 & \\
\hline Branca & $21(44,7)$ & 25,0 & 45,0 & 30,0 & \\
\hline Escolaridade (anos) & & & & & 0,024 \\
\hline$\geq 11$ & $21(44,7)$ & 4,8 & 52,4 & 42,9 & \\
\hline$\leq 10$ & $26(55,3)$ & 48,0 & 20,0 & 32,0 & \\
\hline Trabalho remunerado & & & & & 0,171 \\
\hline Sim & $27(57,4)$ & 19,2 & 38,5 & 42,3 & \\
\hline Não & $20(42,6)$ & 40,0 & 30,0 & 30,0 & \\
\hline Nível Socioeconômico & & & & & 0,792 \\
\hline De $A$ a B & $3(6,4)$ & 33,3 & 33,3 & 33,3 & \\
\hline DeCaD & $32(68,1)$ & 22,6 & 38,7 & 38,7 & \\
\hline $\mathrm{E}$ & $12(25,5)$ & 41,7 & 25,0 & 33,3 & \\
\hline
\end{tabular}

* GPG - Ganho de Peso Gestacional; ** Valor em negrito é estaticamente significativo $(p \leq 0,05)$. 
Tabela 2 - Variáveis obstétricas e de medidas antropométricas referentes ao primeiro trimestre gestacional em relação à prevalência de ganho de peso gestacional em gestantes atendidas na Atenção Básica de Saúde de Caxias do Sul, RS. 2017 ( $n=47)$

\begin{tabular}{|c|c|c|c|c|c|}
\hline Variáveis de exposição & n (\%) & $\begin{array}{c}\text { Prevalência } \\
\text { de GPG* } \\
\text { Insuficiente }\end{array}$ & $\begin{array}{c}\text { Prevalência de } \\
\text { GPG* }^{*} \\
\text { Adequado }\end{array}$ & $\begin{array}{c}\text { Prevalência de } \\
\text { GPG* } \\
\text { Excessivo }\end{array}$ & p-valor** \\
\hline Histórico de aborto & & & & & 0,056 \\
\hline Não & $38(80,9)$ & 21,1 & 39,5 & 39,5 & \\
\hline Sim & $9(19,1)$ & 62,5 & 12,5 & 25,0 & \\
\hline Gestações anteriores & & & & & 0,080 \\
\hline 3 ou mais & $6(12,8)$ & 80,0 & 20,0 & 0,0 & \\
\hline De 1 a 2 & $25(53,2)$ & 24,0 & 32,0 & 44,0 & \\
\hline Nenhuma & $16(34,0)$ & 18,8 & 43,8 & 37,5 & \\
\hline IMC pré-gestacional & & & & & 0,975 \\
\hline Eutróficas & $25(53,2)$ & 25,0 & 41,7 & 33,3 & \\
\hline Não Eutróficas & $22(46,8)$ & 31,8 & 27,3 & 32,0 & \\
\hline
\end{tabular}

anos ou mais de estudo apresentaram maior prevalência de GPG adequado $(52,4 \%)$ ( $p$-valor 0,024$)$ (Tabela 1).

A Tabela 2 apresenta as variáveis obstétricas e antropométricas referentes ao primeiro trimestre gestacional. Pode-se observar que $19,1 \%$ apresentaram histórico de aborto, 53,2\% tiveram de uma a duas gestações anteriores e $46,8 \%$ foram classificadas como não eutróficas segundo o IMC pré-gestacional.

Referente ao GPG, os resultados do estudo mostraram que gestantes com histórico de aborto apresentaram maior prevalência de GPG insuficiente $(62,5 \%)$ ( $p$-valor 0,056$)$, bem como observa-se uma maior prevalência de GPG insuficiente naquelas com 3 ou mais gestações anteriores $(80,0 \%)$ e maior prevalência de GPG adequado entre as gestantes com nenhuma gestação anterior $(43,8 \%)$ ( $p$-valor 0,080$)$. Ressalta-se, entretanto, que ambas as variáveis não apresentaram significância estatística (Tabela 2).

A Tabela 3 apresenta as variáveis de estilo de vida e comportamentais referentes ao terceiro trimestre gestacional. Destaca-se que $89,4 \%$ das gestantes ressaltam não ter o hábito de realizar atividade física, $76,6 \%$ têm o hábito de realizar café da manhã e $66,0 \%$ afirmam ter o hábito de beliscar entre as refeições. Em relação às variáveis comportamentais, os resultados demonstraram que $15,2 \%$ das gestantes apresentaram insatisfação corporal e $52,2 \%$ foram classificadas com sintomas depressivos de moderados a severos.
Em relação ao hábito de beliscar entre as refeições, observa-se que estas gestantes obtiveram elevada prevalência de GPG excessivo $(45,0 \%)$ ( $p$-valor $0,118)$, embora esta variável não tenha apresentado significância estatística (Tabela 3).

A Tabela 4 descreve as variáveis relacionadas ao nascimento. Nela observa-se que $85,1 \%$ dos bebês nasceram termo. Em relação ao Apgar, 97,8\% apresentaram 8 pontos ou mais. É possível identificar, entretanto, que $21,3 \%$ dos recém-nascidos apresentaram baixo peso, $72,3 \%$ e $6,4 \%$ peso normal e macrossomia, respectivamente. Referente ao comprimento ao nascer, $66 \%$ nasceram com $48 \mathrm{~cm}$ ou mais. Além disso, os dados demonstraram que $61,7 \%$ nasceram de parto normal, $85,1 \%$ dos recém-nascidos não apresentaram internações e $74,5 \%$ receberam AME na primeira semana de vida.

Quanto aos dados do nascimento, identificou-se associação significativa com o comprimento ao nascer ( $p$-valor 0,035 ), quando os bebês que nasceram com $47 \mathrm{~cm}$ ou menos apresentaram maior prevalência de GPG insuficiente $(45,0 \%)$ e os que nasceram com $48 \mathrm{~cm}$ ou mais apresentaram elevada prevalência de GPG excessivo (46,2\%). Ainda, é possível observar associação significativa com a alimentação na primeira semana de vida ( $p$-valor 0,037), em que o AME apresentou maior prevalência em gestantes com GPG excessivo e Aleitamento Misto/Fórmulas Infantis/Outros demonstrou maior prevalência de GPG adequado (Tabela 4). 
Tabela 3 - Variáveis de estilo de vida e comportamentais referentes ao terceiro trimestre gestacional em relação à prevalência de ganho de peso gestacional em gestantes atendidas na Atenção Básica de Saúde de Caxias do Sul, RS. 2017 (n=47)

\begin{tabular}{|c|c|c|c|c|c|}
\hline Variáveis de exposição & n (\%) & $\begin{array}{l}\text { Prevalência de } \\
\text { GPG* Insuficiente }\end{array}$ & $\begin{array}{l}\text { Prevalência de } \\
\text { GPG* Adequado }^{*}\end{array}$ & $\begin{array}{l}\text { Prevalência de } \\
\text { GPG* Excessivo }\end{array}$ & p-valor** \\
\hline Atividade física & & & & & 0,134 \\
\hline Sim & $5(10,6)$ & 0,0 & 40,0 & 60,0 & \\
\hline Não & $42(89,4)$ & 31,7 & 34,1 & 34,1 & \\
\hline Realiza Café da Manhã & & & & & 0,203 \\
\hline Sim & $36(76,6)$ & 24,3 & 35,1 & 40,5 & \\
\hline Não & $11(23,4)$ & 44,4 & 33,3 & 22,2 & \\
\hline Beliscar entre as refeições & & & & & 0,118 \\
\hline Não & $16(34,0)$ & 38,5 & 30,8 & 30,8 & \\
\hline Sim & $31(66,0)$ & 15,0 & 40,0 & 45,0 & \\
\hline Insatisfação corporal & & & & & 0,684 \\
\hline Ausência & $39(84,8)$ & 25,7 & 37,1 & 37,1 & \\
\hline Presença & $7(15,2)$ & 36,4 & 27,3 & 36,4 & \\
\hline Sintomas depressivos & & & & & 0,687 \\
\hline Ausência & $6(13,0)$ & 16,7 & 33,3 & 50,0 & \\
\hline Leve & $16(34,8)$ & 31,3 & 37,5 & 31,3 & \\
\hline Moderada à severa & $24(52,2)$ & 29,2 & 33,3 & 37,5 & \\
\hline
\end{tabular}

* GPG - Ganho de Peso Gestacional; ** Valores em negrito são estaticamente significativos $(p \leq 0,05)$.

Tabela 4 - Variáveis relacionadas ao nascimento em relação à prevalência de ganho de peso gestacional em gestantes atendidas na Atenção Básica de Saúde de Caxias do Sul, RS. 2017 (n=47)

\begin{tabular}{|c|c|c|c|c|c|}
\hline Variáveis de exposição & n (\%) & $\begin{array}{c}\text { Prevalência de } \\
\text { GPG* Insuficiente }\end{array}$ & $\begin{array}{l}\text { Prevalência de } \\
\text { GPG* Adequado }\end{array}$ & $\begin{array}{l}\text { Prevalência de } \\
\text { GPG* Excessivo }\end{array}$ & p-valor** \\
\hline Semana gestacional do nascimento & & & & & 0,068 \\
\hline Termo & $40(85,1)$ & 25,6 & 30,8 & 43,6 & \\
\hline Pré-termo & $7(14,9)$ & 42,9 & 57,1 & 0,0 & \\
\hline Apgar & & & & & 0,153 \\
\hline$\leq 7$ pontos & $1(2,2)$ & 100,0 & 0,0 & 0,0 & \\
\hline$\geq 8$ pontos & $45(97,8)$ & 22,6 & 41,9 & 35,5 & \\
\hline Peso ao nascer & & & & & 0,100 \\
\hline Baixo peso & $10(21,3)$ & 40,0 & 50,0 & 10,0 & \\
\hline Normal & $34(72,3)$ & 24,2 & 33,3 & 42,4 & \\
\hline Macrossomia & $3(6,4)$ & 33,3 & 0 & 66,7 & \\
\hline Comprimento & & & & & 0,035 \\
\hline$\leq 47 \mathrm{~cm}$ & $16(34,0)$ & 45,0 & 30,0 & 25,0 & \\
\hline$\geq 48 \mathrm{~cm}$ & $31(66,0)$ & 15,4 & 38,5 & 46,2 & \\
\hline Perímetro cefálico & & & & & 0,542 \\
\hline$\leq 33 \mathrm{~cm}$ & $20(42,6)$ & 31,6 & 36,8 & 31,6 & \\
\hline$\geq 34 \mathrm{~cm}$ & $27(57,4)$ & 25,9 & 33,3 & 37,0 & \\
\hline Tipo de parto & & & & & 0,560 \\
\hline Parto Normal & $29(61,7)$ & 21,4 & 42,9 & 35,7 & \\
\hline Cesárea & $18(38,3)$ & 38,9 & 22,2 & 38,9 & \\
\hline Internação do RN & & & & & 0,187 \\
\hline Não & $40(85,1)$ & 25,6 & 33,3 & 41,0 & \\
\hline $\operatorname{Sim}$ & $7(14,9)$ & 42,9 & 42,9 & 14,3 & \\
\hline $\begin{array}{l}\text { Alimentação na primeira semana de } \\
\text { vida }\end{array}$ & & & & & 0,037 \\
\hline Aleitamento Materno Exclusivo & $35(74,5)$ & 25,3 & 29,4 & 47,1 & \\
\hline $\begin{array}{l}\text { Aleitamento Misto/Fórmulas/ } \\
\text { Outros }\end{array}$ & $12(25,5)$ & 41,7 & 50,0 & 8,3 & \\
\hline
\end{tabular}

* GPG - Ganho de Peso Gestacional; ** Valores em negrito são estaticamente significativos $(p \leq 0,05)$. 


\section{DISCUSSÃO}

O presente estudo teve como objetivo medir o GPG e identificar os fatores associados em gestantes e recém-nascidos de Caxias do Sul/RS. Comparando a estudos internacionais, segundo Kominiarek et al. (2018) (Chicago) e Lopez-Cepero et al. (2018) (Massachusetts/Boston), observou-se elevadas prevalências de GPG excessivo ( $46 \%$ e $62,8 \%$, respectivamente) entre as populações, as quais encontram-se maiores que no presente estudo. Em relação aos estudos em âmbito nacional, segundo Souza et al. (2017) (Minas Gerais/Brasil), observou-se maior prevalência para o GPG insuficiente e adequado ( $41 \%$ e $39,0 \%$ ). Visualizando os resultados é possível observar que as prevalências do GPG se modificam de acordo com a população estudada, e, ainda assim, as prevalências apresentam-se maiores quando comparadas ao presente estudo. Conforme artigos regionais, realizados em Pelotas/ RS e Porto Alegre/RS, entretanto, observaram-se elevadas prevalências para o GPG adequado e excessivo (36\% adequado, $25,5 \%$ excessivo e $33,6 \%$ adequado e $38 \%$ excessivo) (CASTILLO; SANTOS; MATIJASEVICH, 2016; MASTELLA et al., 2018). Desse modo, percebe-se que os achados se assemelham aos do presente estudo e pode-se observar uma elevada prevalência de GPG adequado entre as populações. Destaca-se, todavia, a importância de atentar para o GPG excessivo nas Regiões do Sul do Brasil, sabendo-se que está associado a complicações durante a gestação, entre elas a maior probabilidade de desenvolver Diabetes Mellitus Gestacional (DMG), ter recém-nascido macrossômico e a maior taxa de cesarianas (ORNAGHI et al., 2018).

Quanto aos fatores associados ao GPG, observa-se associação significativa com a escolaridade, quando as gestantes com 11 anos ou mais de estudo apresentaram maior prevalência de GPG adequado e as com até 10 anos de estudo maior prevalência de GPG insuficiente. Na China, as gestantes com menor escolaridade apresentaram maior GPG (HUANG et al., 2018). Por outro lado, no Rio de Janeiro, a baixa escolaridade foi associada ao GPG insuficiente nas gestantes (FRAGA; THEME FILHA, 2014). Ainda assim, segundo estudo realizado em seis cidades brasileiras, é possível observar que as gestantes com baixa escolaridade apresentaram maior GPG insuficiente e as gestantes com maior escolaridade apresentaram maior GPG excessivo (DREHMER et al., 2013). Sugere-se que a escolaridade seja um fator determinante do GPG e acredita-se que a baixa escolaridade afete o acesso às informações. Desta forma, acredita-se que a elevada escolaridade facilite o acesso às informações, bem como o acesso à alimentação adequada, possibilitando o GPG adequado e facilitando o GPG excessivo.

Observa-se que a as gestantes com menos idade apresentaram maior GPG excessivo, embora esta variável não tenha apresentado significância estatística. Segundo achados de Drehmer et al. (2013), as gestantes mais novas apresentam maior GPG excessivo comparando as de mais idade, assemelhando-se aos achados do presente estudo. Ainda, estudo mostra que gestantes com mais idade apresentam maior possibilidade de apresentar GPG insuficiente, enquanto as gestantes mais jovens GPG excessivo (FRAGA; THEME FILHA, 2014). Sugere-se que mulheres com mais idade apresentam maior possibilidade de GPG excessivo, podendo estar associado a mudanças fisiológicas que geram um aumento nos riscos para a saúde da gestante e do feto.

Sabe-se que a classe social mais elevada está associada ao GPG adequado (ONWUKA et al., 2017). O nível socioeconômico, entretanto, aparenta ser um reflexo do acesso a informações, este diretamente relacionado à maior escolaridade (O'BRIEN; ALBERDI; MCAULIFFE, 2017). Assim, pode-se explicar a elevada prevalência de GPG insuficiente $(41,7 \%)$ em gestantes de baixo nível socioeconômico, embora esta variável não apresente significância estatística no presente estudo.

O histórico de aborto é fator de risco para prematuridade entre gestantes (ZHONG; CUI, 2014). Em Aragão, Espanha, o GPG insuficiente foi associado ao histórico de aborto (RAMÓN-ARBUÉS; MARTÍNEZ ABADÍA; MARTíN GÓMEZ, 2017); achados estes que corroboram com o presente estudo, em que as gestantes com histórico de aborto apresentaram maior prevalência de GPG insuficiente (62\%), embora não apresentem significância estatística. Sugere-se a realização de estudos voltados a explicar a influência do histórico de aborto na inadequação do GPG.

Quanto à atividade física, observa-se que praticar 150 minutos ou mais por semana apresentou elevada prevalência (60\%) de GPG excessivo. Segundo estudo, no entanto, mulheres com IMC pré-gestacional mais alto e baixa atividade física mostraram duas vezes mais chances de ter GPG excessivo (YONG et al., 2016). Sabe-se que intervenções comportamentais que melhorem a alimentação, juntamente com a prática de atividade física, atuam no controle do GPG (GALLAGHER et al., 2018). Sendo assim, acredita-se que a atividade física contribua para a manutenção 
e adequação do GPG, entretanto esta deverá ser associada a uma alimentação adequada, a qual não foi controlada no presente estudo.

Referente ao hábito de beliscar entre as refeições, encontrou-se uma maior prevalência de GPG excessivo (45\%). Ainda assim, comer lanches frequentemente está associado ao GPG excessivo (CHEN et al., 2018). Sabe-se, porém, que a alimentação consciente desempenha um papel no comportamento alimentar, bem como a atenção plena ao se alimentar, atuando diretamente no controle do GPG (HUTCHINSON et al., 2017). Sugere-se que as gestantes com o hábito de beliscar entre as refeições acabam por ingerir uma quantidade maior de alimentos e calorias, além de, possivelmente, não estarem realizando uma alimentação consciente e com a atenção plena recomendada, facilitando, assim, o GPG excessivo.

Em relação aos fatores associados ao recém-nascido, o presente estudo encontrou associação significativa com o comprimento ao nascer, no qual as gestantes com GPG insuficiente tiveram bebês menores. Na Itália, o GPG insuficiente em gestantes com IMC pré-gestacional normal foi associado ao feto pequeno para a idade gestacional no nascimento (ORNAGHI et al., 2018). Considerando-se que os achados na literatura se assemelham aos do presente estudo, acredita-se que o GPG insuficiente possibilite o nascimento de recém-nascidos pequenos para a idade gestacional e com menor comprimento, aumentando os riscos para a saúde do bebê.

A alimentação na primeira semana de vida também apresentou associação significativa com o desfecho. As gestantes com GPG adequado e insuficiente ressaltaram maior prevalência para o aleitamento misto, com fórmulas infantis ou outros; já as gestantes com GPG excessivo apresentaram maior prevalência de AME. Sabe-se, entretanto, que o GPG insuficiente aumenta os riscos para o insucesso no início do aleitamento materno (WINKVIST et al., 2015). Além disso, sabe-se que as gestantes com sobrepeso e obesidade apresentam probabilidade aumentada de desmame precoce ( 3 meses de idade) (CASTILLO; SANTOS; MATIJASEVICH, 2016), e que o aleitamento materno apresenta efeito protetor contra o excesso de peso tanto para a mãe quanto para a criança (MASTROENI et al., 2017). Sugere-se que nesta população o GPG insuficiente aumente o risco para o insucesso do aleitamento materno na primeira semana pós-parto e o GPG excessivo possibilite o AME na primeira semana de vida, mas não se descarta a possibilidade de que esse dado seja influenciado pela falta de instrução sobre o aleitamento materno durante a gestação.
Quanto ao Apgar em 5 minutos, o GPG adequado apresentou maior prevalência de Apgar maior ou igual a 8 pontos, enquanto o GPG insuficiente de menor ou igual a 7 pontos, embora não tenha significância estatística. O GPG excessivo em mulheres com hipertensão crônica está associado ao Apgar em 5 minutos menor que 7 pontos, o que facilita o aumento de internações aos recém-nascidos (YEE; CAUGHEY; CHENG, 2017). Estudos demonstram que o GPG excessivo está associado a menores pontuações de Apgar (GAVARD, 2017). Por outro lado, estudos não encontraram associação suficientemente clara entre o GPG e o Apgar (HIROOKA-NAKAMA et al., 2018). Desta forma, acredita-se que o GPG adequado desempenha papel de proteção, favorecendo melhores escores de Apgar em recém-nascidos.

No presente estudo não foram encontradas associações significativas entre o GPG e o peso ao nascer. Observa-se, todavia, que a macrossomia foi mais presente nas gestantes com GPG excessivo. Na Itália, a maior probabilidade de baixo peso ao nascer foi associada a gestantes obesas e ao GPG abaixo do recomendado ( $\mathrm{NUCCl}$ et al., 2018). Além disso, sabe-se que o excesso de peso pré-gestacional e o GPG excessivo estão associados ao recém-nascido grande para a idade gestacional e macrossômicos, posto que o GPG adequado atua como proteção para recém-nascidos macrossômicos (HIROOKA-NAKAMA et al., 2018; NU$\mathrm{CCl}$ et al., 2018). Ainda, estudo demonstra que o GPG insuficiente foi associado a recém-nascidos pequenos para a idade gestacional (ORNAGHI et al., 2018). Acredita-se que o GPG adequado, juntamente com o IMC eutrófico e uma alimentação adequada, possibilite um peso ao nascer normal e atue como proteção para o baixo peso e macrossomia.

No que se refere à ocorrência de internação por mais de 72 horas nos recém-nascidos, observa-se que o GPG excessivo apresentou menor prevalência $(14,3 \%)$. Ao mesmo tempo, não houve diferenças entre as prevalências de ocorrência de internação para GPG adequado ou insuficiente. Ainda assim, segundo estudo, as gestantes com GPG excessivo apresentam maiores taxas de internação em Unidade de Terapia Intensiva Neonatal (Utin) (YEE; CAUGHEY; CHENG, 2017). Nos Estados Unidos, o GPG excessivo aumentou o tempo de internação do recém-nascido, entretanto o GPG insuficiente aumentou a ocorrência de interações em Utin (BAUGH et al., 2016). Por isso, sugere-se que o GPG insuficiente e excessivo possivelmente afete o tempo de internação para os recém-nascidos, enquanto o GPG adequado atue como um 
fator de proteção, tanto para o tempo de internação quanto para outros desfechos negativos referente à saúde do recém-nascido.

Como limitação deste estudo, destaca-se o pequeno número amostral, quando ocorreu uma grande porcentagem de perdas $(38,1 \%)$. O possível viés de memória, em virtude dos questionários, nos quais utilizou-se perguntas retroativas, pode vir a confundir a gestante. Ainda assim, percebe-se que são limitados os estudos nacionais e regionais que acompanharam o GPG e relacionaram os fatores de risco gestacionais e os do recém-nascidos.

$O$ presente estudo apresenta, contudo, como potenciais, ter gestantes de diferentes regiões do munícipio incluídas no estudo e os dados coletados; assim, obteve-se uma grande e diversificada quantidade de informações relevantes. Além disso, destaca-se o desenho do estudo, tratando-se de um estudo longitudinal, permitindo o acompanhamento durante a gestação e a possibilidade de identificar a evolução de questões relacionadas à saúde da gestante ao longo do período gestacional, e, ainda, o controle, precaução e responsabilidade para o tratamento dos dados, minimizando erros de digitação. Todos os participantes do estudo receberam treinamento e foram capacitados para realizar a coleta, aumentando a confiança perante os dados coletados e os resultados obtidos no presente estudo.

\section{CONCLUSÕES}

Conclui-se que a população investigada apresenta elevada prevalência de GPG excessivo. Ainda, identificou-se associação significativa entre o GPG insuficiente com a baixa escolaridade da gestante e ao menor comprimento ao nascer. A alimentação na primeira semana de vida do recém-nascido foi associada ao GPG excessivo, no qual houve maior prevalência para AME. Os achados são relevantes, podendo ocasionar problemas para a saúde do recém-nascido. Desta forma, percebe-se a necessidade de estratégias para adequar e orientar o GPG, assim como instruir hábitos de vida saudáveis e reforçar a necessidade do cuidado pré-natal para a saúde da gestante e do recém-nascido.

\section{REFERÊNCIAS}

BAUGH, N. et al. The impact of maternal obesity and excessive gestational weight gain on maternal and infant outcomes in Maine: Analysis of pregnancy risk assessment monitoring system results from 2000 to 2010. Journal of pregnancy, v. 2.016, p. 1-10, June 2016.
BRASIL. Ministério da Saúde. Secretaria de Atenção à Saúde. Departamento de Ações Programáticas Estratégicas. Atenção à saúde do recém-nascido: guia para os profissionais de saúde. 2. ed. atual. Brasília: Ministério da Saúde, 2011. Disponível em: http://bvsms.saude.gov.br/bvs/publicacoes/atencao_saude_recem_nascido_v1.pdf. Acesso em: 20 maio 2018.

CASTILLO, H.; SANTOS, I. S.; MATIJASEVICH, A. Maternal pre-pregnancy $\mathrm{BMI}$, gestational weight gain and breastfeeding. European journal of clinical nutrition, v. 70, n. 4, p. 431, Jan. 2016.

CHEN, Y. Y. et al. Eating Frequency and Gestational Weight Gain and Its Association with Birth Body Mass of Neonates: a Birth Cohort Study. Sichuan da xue xue bao. Yi xue ban = Journal of Sichuan University. Medical science edition, v. 49, n. 2, p. 258-263, Mar. 2018.

CORDÁS, T. A.; CASTILHO, S. Imagem corporal nos transtornos alimentares: instrumento de avaliação: Body Shape Questionnaire. Psiquiatria Biológica, v. 2, n. 1, p. 17-21, 1994.

COUNCIL, N. R. Weight gain during pregnancy: reexamining the guidelines. National Academies Press. Disponível em: https://www.ncbi.nlm.nih.gov/pubmed/20669500. 2009. Acesso em: 8 June 2018.

DREHMER, M. et al. Association of second and third trimester weight gain in pregnancy with maternal and fetal outcomes. PloS One, v. 8, n. 1, p. e54704, Jan. 2013.

FRAGA, A. C. S. A.; THEME FILHA, M. M. Factors associated with gestational weight gain in pregnant women in Rio de Janeiro, Brazil, 2008. Cadernos de Saúde Pública, v. 30, n. 3, p. 633-644, Mar. 2014.

GALLAGHER, D. et al. Greater Neonatal Fat Free Mass and Similar Fat Mass Following a Randomized Trial to Control Excess Gestational Weight Gain. Obesity, v. 26, n. 3, p. 578587, Feb. 2018.

GAVARD, J. A. Gestational weight gain and maternal and neonatal outcomes in underweight pregnant women: a population-based historical cohort study. Maternal and Child Health Journal, v. 21, n. 5, p. 1.203-1.210, 2017.

HIROOKA-NAKAMA, J. et al. Optimal weight gain in obese and overweight pregnant Japanese women. Endocrine journal, v. 65, n. 5, p. 557-567, Feb. 2018.

HUANG, X. et al. Gestational weight gain in Chinese women-results from a retrospective cohort in Changsha, China. BMC pregnancy and childbirth, v. 18, n. 1, p. 185, May 2018.

HUTCHINSON, A. D. et al. Understanding maternal dietary choices during pregnancy: The role of social norms and mindful eating. Appetite, v. 112, p. 227-234, May 2017.

KOMINIAREK, M. A. et al. Stress during pregnancy and gestational weight gain. Journal of Perinatology, v. 38, p. 462467, Jan. 2018.

LOPEZ-CEPERO, A. et al. Association Between Obstetric Provider's Advice and Gestational Weight Gain. Maternal and Child Health Journal, v. 22, n. 8, p. 1.127-1.134, Aug. 2018. 
MASTELLA, L. S. et al. Influence of maternal weight gain on birth weight: a gestational diabetes cohort. Archives of endocrinology and metabolism, v. 62, n. 1, p. 55-63, Feb. 2018. MASTROENI, M. F. et al. Breast-feeding duration for the prevention of excess body weight of mother-child pairs concurrently: a 2-year cohort study. Public health nutrition, v. 20, n. 14, p. 2.537-2.548, Oct. 2017.

NUCCl, D. et al. Pre-pregnancy body mass index, gestational weight gain and adverse birth outcomes: some evidence from Italy. Annali di Igiene: Medicina Preventiva e di Comunita, v. 30, n. 2, p. 140-152, Jan. 2018.

O'BRIEN, E. C.; ALBERDI, G.; MCAULIFFE, F. M. The influence of socioeconomic status on gestational weight gain: a systematic review. Journal of Public Health, v. 40, n. 1, p. 41-55, Mar. 2017.

ONWUKA, C. I. et al. Patterns of gestational weight gain and its association with birthweight in Nigeria. Nigerian Journal of Clinical Practice, v. 20, n. 6, p. 754-760, June 2017.

ORNAGHI, S. et al. Impact of excessive pre-pregnancy body mass index and abnormal gestational weight gain on pregnancy outcomes in women with chronic hypertension. Pregnancy hypertension, v. 12, p. 90-95, Apr. 2018.

RAMÓN-ARBUÉS, E.; MARTÍNEZ ABADÍA, B.; MARTÍN GÓMEZ, S. Ganancia de peso gestacional y retención de peso posparto en una cohorte de mujeres en Aragón (España). Nutricion Hospitalaria, v. 34, n. 5, Oct. 2017.

SOUZA, L. A. et al. Could physical discomforts be related to weight gain and parity in last trimester pregnant women? Revista Dor, v. 18, n. 1, p. 18-22, Mar. 2017.

VOIDAZAN, S. et al. Associations between lifestyle factors and smoking status during pregnancy in a group of Romanian women. Birth defects research, v. 110, p. 519-526, Jan. 2018.
WEISSGERBER, T. L.; WOLFE, L. A. Physiological adaptation in early human pregnancy: adaptation to balance maternal-fetal demands. Applied physiology, nutrition, and metabolism, v. 31, n. 1, p. 1-11, Jan. 2006.

WHO. World Health Organization. Global recommendations on physical activity for health. Geneva: World Health Organization, 2010. Disponível em: https://www.who.int/ dietphysicalactivity/global-PA-recs-2010.pdf. Acesso em: 10 May 2018.

WINKVIST, A. et al. Maternal Prepregnant Body Mass Index and Gestational Weight Gain Are Associated with Initiation and Duration of Breastfeeding among Norwegian Mothers-3. The Journal of Nutrition, v. 145, n. 6, p. 1.2631.270, Apr. 2015.

YEE, L. M.; CAUGHEY, A. B.; CHENG, Y. W. Association between gestational weight gain and perinatal outcomes in women with chronic hypertension. American Journal of Obstetrics \& Gynecology, v. 217, n. 3, p. 348.e1-348.e9, Sept. 2017.

YONG, H. Y. et al. Prepregnancy body mass index, height and physical activity are associated with rate of gestational weight gain among Malaysian mothers. Journal of Obstetrics and Gynaecology Research, v. 42, n. 9, p. 1.094-1.101, May 2016.

ZHONG, X. Q.; CUI, Q. L. Comparative analysis of risk factors for preterm and small-for-gestational-age births. Zhongguo dang dai er ke za zhi=Chinese Journal of Contemporary Pediatrics, v. 16, n. 12, p. 1.202-1.205, Dec. 2014. 